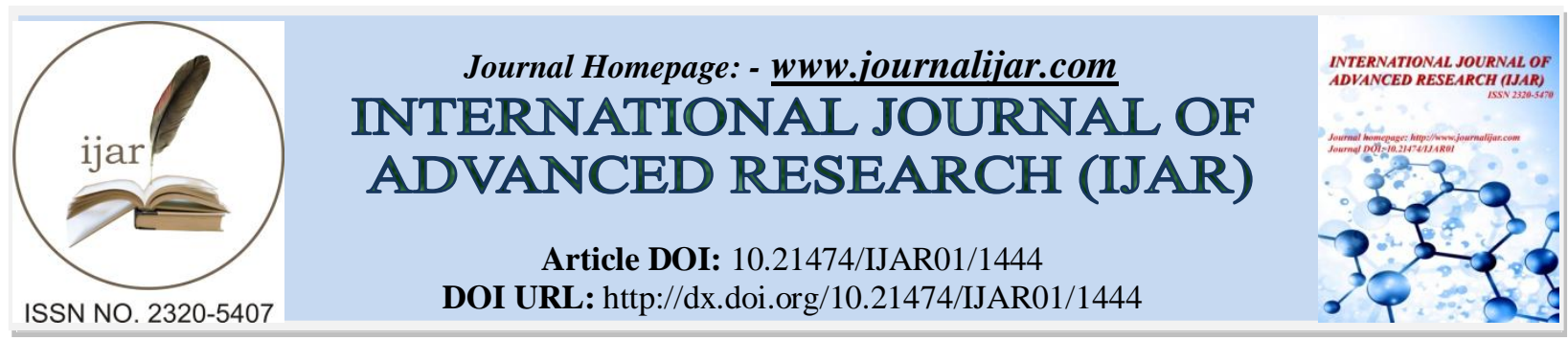

RESEARCH ARTICLE

\title{
NUMERICAL AND APPROXIMATE ANALYTIC SOLUTION OF MHD VISCOELASTIC NANOFLUID FLOW OVER A TWO WAY STRETCHING /SHRINKING SHEET.
}

M. Satyakrishna ${ }^{1}$ and Achala. L. Nargund ${ }^{2}$.

1. Department of Mathematics, MES Degree College, Malleswaram, Bangalore.

2. Post Graduate Department of Mathematics, MES Degree College, Malleswaram, Bangalore.

\section{Manuscript Info}

Manuscript History

Received: 12 June 2016

Final Accepted: 19 July 2016

Published: August 2016

Key words:-

Viscoelastic nanofluid, Shrinking sheet, Stretching sheet, Brownian motion, MHD flow, Runge Kutta Merson method, Homotopy Analysis Method.

\begin{abstract}
We analyze the effect of magnetic field on the flow and heat transfer of non-Newtonian nanofluid over a Two way stretching sheet, where magnetic field is orientated normally to the plate. The Brownian motion [1] and Thermophoresis effects are also considered. The boundary layer equations governed by the partial differential equations are transformed into a set of ordinary differential equations with the help of local similarity transformations. The differential equations are solved by Homotopy Analysis Method (HAM) [2] and Runge Kutta Merson method (RKM) [3, 4, 5]. We have drawn the graphs for velocity distribution for HAM solution and RKM solution.
\end{abstract}

\section{Introduction:-}

Nanofluids have enormous industrial, transportation, electronics, biomedical applications, such as in advanced nuclear systems, cylindrical heat pipes, automobiles, fuel cells, drug delivery, biological sensors, and hybridpowered engines. Nanofluids are fluids with suspended nanoparticles with average sizes of 1-100 nm. The nanoparticles are typically made of oxides such as alumina, silica, titania and copper oxide, carbides, and metals such as copper and gold. Carbon nanotubes and diamond nanoparticles have also been used in nanofluids. The base fluid is usually a conventional heat transfer fluid, such as oil, water, and ethylene glycol. Other base fluids are biofluids, polymer solutions, and some lubricants.

In some practical problems such as the magnetohydrodynamic (MHD) generators, pumps, enhanced oil recovery, thermal insulators, electronic packages, and cooling of nuclear reactor, the flow of electrically conducting fluid occurs in the presence of a transverse magnetic field. Many fluids of industrial and geophysical importance are nonNewtonian. In real situations in nanofluids, the base fluid does not satisfy the properties of Newtonian fluids; hence, it is more justified to consider them as viscoelastic fluids; for example, ethylene glycol- $\mathrm{Al}_{2} \mathrm{O}_{3}$, ethylene glycol-CuO, and ethylene glycol- $\mathrm{ZnO}$ are some examples of viscoelastic nanofluids.

The term nanofluids (nanoparticle fluid suspensions) was coined by Choi [6] in 1995 to describe this new class of nanotechnology-based heat transfer in fluids. A comprehensive survey of convective transport in nanofluids was made by Buongiorno [7]. He explained the significance of the Brownian diffusion and the thermophoretic diffusion of the nanoparticles. Khan and Pop [8] have used the model of Kuznetsov and Nield [9] to study the boundary layer 
flow of nanofluid over a stretching sheet. Makinde and Aziz [10] extended the work of Khan and Pop [8] for convective boundary conditions.

The MHD boundary layer flow of an incompressible and electrically conducting viscoelastic fluid past a linear stretching sheet was studied by Subhas Abel et al. [11]. The momentum and heat transfer characteristics of the boundary layers of an incompressible electrically conducting fluid flow of a viscoelastic fluid over a stretching sheet are investigated by Prasad et al. [12, 13]. Recently, Hameed et al. [14] reported a similarity solution for MHD free convection heat generation flow over a vertical semi-infinite flat plate in the case of nanofluids.

McCormack and Crane [15] have provided comprehensive discussion on boundary layer flow caused by stretching of an elastic flat sheet moving in its own plane with a velocity varying linearly with distance. P. S. Gupta and A. S. Gupta [16] and Dutta et al. [17] extended the work of McCormack and Crane [15] by including the effects of heat and mass transfer under different situations. Wang $[18,19]$ discussed the partial slip effects on the planar stretching flow. Noghrehabadi et al. [20] investigated the development of the slip effects on the boundary layer flow and heat transfer over a stretching sheet.

Rana and Bhargava [21] have studied the heat transfer characteristic in the mixed convection flow of a nanofluid along a vertical plate with heat source/sink. Mania Goyal and Rama Bhargav (2014) [22] have extended the work of Noghrehabadi et al. [20] by taking base fluid as second-grade fluid and have obtained numerical solution by using finite element method.

In the present paper, we analyze the effect of magnetic field on the flow of non-Newtonian nanofluid over a two way shrinking/stretching sheet, where magnetic field is orientated normally to the plate. The boundary layer equations governed by the partial differential equations are transformed into a set of ordinary differential equations with the help of local similarity transformations. The differential equations are solved by Homotopy Analysis Method (HAM) and Runge Kutta Merson method (RKM). We have examined the effects of different controlling parameters, namely, the Brownian motion parameter, uniform magnetic field, viscoelastic parameter, Prandtl number, and Lewis number on the flow.

\section{Mathematical formulation:-}

Consider two dimensional flow of an incompressible, non-Newtonian, nano fluid flowing steadily under the effect of external magnetic field applied normally to the flow over a shrinking/stretching sheet. The $\mathrm{x}$-axis is taken along the plate, y-axis perpendicular to it. Temperature and concentrations over the plate are maintained uniform. Fluid and nano particles are assumed to be in thermal equilibrium. The pressure gradient and external forces are absent. The following are the reduced governing equations of the considered problem

$$
\begin{aligned}
& f^{\prime \prime \prime}+f f^{\prime \prime}-f^{\prime 2}-\alpha\left(f^{\prime \prime 2}-2 f^{\prime} f^{\prime \prime \prime}+f^{i v}\right)-M f^{\prime}=0, \\
& \frac{1}{\operatorname{Pr}} \theta^{\prime \prime}+f \theta^{\prime}+N b \theta^{\prime} \varphi^{\prime}+N t \theta^{\prime 2}=0, \\
& \varphi^{\prime \prime}+\operatorname{Lef} \varphi^{\prime}+\frac{N t}{N b} \theta^{\prime \prime}=0,
\end{aligned}
$$

where primes denote differentiation with respect to $\eta$ and $\operatorname{Pr}, \mathrm{Le}, \mathrm{Nb}, \mathrm{Nt}$ and $\alpha$ are Prandtle number, Lewis number, Brownian motion parameter, Thermophoresis parameter and viscoelastic parameter respectively. The boundary conditions are

$$
\begin{aligned}
& f(0)=0, f^{\prime}(0)=1+K f^{\prime \prime}(0), \theta(0)=1, \varphi(0)=1, \\
& f^{\prime}(\infty)=0, \theta(\infty)=0, \varphi(\infty)=0 .
\end{aligned}
$$

These equations with neglecting temperature are solved by HAM and the obtained solutions are depicted graphically and are observed to match exactly to FEM solutions of Mania Goyal and Rama Bhargava [22].

Numerically we solve these equations by Runge-Kutta Merson Method. 


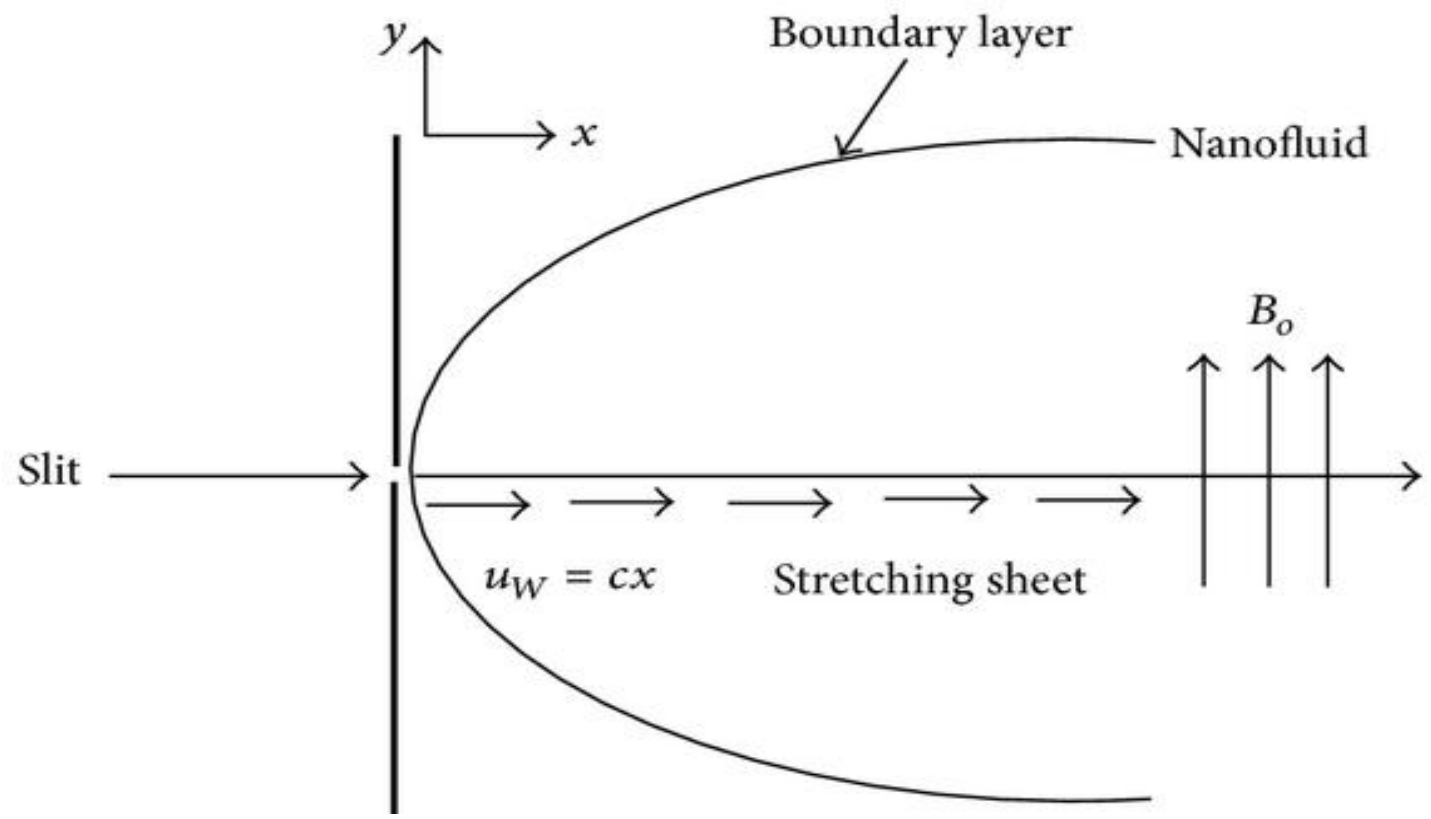

Fig 1:- Geometry of the flow

\section{Method of Solution:-}

Let us consider the following equations and solve by HAM

$$
\begin{aligned}
& f^{\prime \prime \prime}+f f^{\prime \prime}-f^{\prime 2}-\alpha\left(f^{\prime \prime 2}-2 f^{\prime} f^{\prime \prime \prime}+f^{i v}\right)-M f^{\prime}=0, \\
& \frac{1}{\operatorname{Pr}} \theta^{\prime \prime}+f \theta^{\prime}+N b \theta^{\prime} \varphi^{\prime}+N t \theta^{\prime 2}=0, \\
& \varphi^{\prime \prime}+\operatorname{Lef} \varphi^{\prime}+\frac{N t}{N b} \theta^{\prime \prime}=0,
\end{aligned}
$$

The equation (6) is independent of $\theta$ and $\phi$ so first we will solve (6) by homotopy analysis method by neglecting the effect of temperature. Let us choose the auxiliary linear operator as

$$
L=\frac{\partial^{3}}{\partial \eta^{3}}+\frac{\partial^{2}}{\partial \eta^{2}}-M \frac{\partial}{\partial \eta}
$$

Then, we construct a family of partial differential equations as follows $\left.(1-\mathrm{p}) \mathrm{L}\left[\mathrm{F}(\eta, \mathrm{p})-\mathrm{f}_{0}(\eta)\right)\right]=\mathrm{hp}\left\{\frac{\partial^{3} F}{\partial \eta^{3}}+\frac{\partial^{2} F}{\partial \eta^{2}}-\left(\frac{\partial F}{\partial \eta}\right)^{2}-M \frac{\partial F}{\partial \eta}-\alpha\left(\left(\frac{\partial^{2} F}{\partial \eta^{2}}\right)^{2}-2 \frac{\partial F}{\partial \eta} \frac{\partial^{3} F}{\partial \eta^{3}}+\frac{\partial^{4} F}{\partial \eta^{4}}\right)\right\}$

with boundary conditions

$$
\mathrm{F}(0, \mathrm{p})=0, \mathrm{~F}_{\eta}(0, \mathrm{p})=1+\mathrm{F}_{\eta \eta}(0, \mathrm{p}), \mathrm{F}_{\eta}(+\infty, \mathrm{p})=0,
$$

where $\mathrm{F}_{\eta}$ denotes the first-order derivative of $\mathrm{F}(\eta, \mathrm{p})$ with respect to $\eta, \mathrm{p} \in[0,1]$ is the embedding parameter, $\mathbf{h} \neq 0$ is an non zero auxiliary parameter.

We choose the initial guess $\mathrm{f}_{0}(\eta)$ by using linear operator (9) as follows in accordance with boundary conditions (11) and $\mathrm{M}=2$ and $\alpha=0$ as

$$
\mathrm{f}_{0}(\eta)=\frac{4}{3} \mathrm{e}^{\eta}-\frac{1}{3} \mathrm{e}^{-2 \eta},
$$

When $p=0$, we have the solution 


$$
F(\eta, 0)=\mathrm{f}_{0}(\eta)
$$

When $p=1$, we get

$$
F(\eta, 1)=\mathrm{f}(\eta)
$$

Thus as $\mathrm{p}$ increases from 0 to 1 , the solution varies from the initial guess approximation $\mathrm{f}_{0}(\eta)$ to the solution $\mathrm{f}(\eta)$.

Now we will express the solution in terms of Maclaurin series as

$$
F(\eta, p)=F(\eta, 0)+\left.\sum_{k=1}^{+\infty} \frac{p^{k}}{k !} \frac{\partial^{k} F(\eta, p)}{\partial p^{k}}\right|_{p=0},
$$

Let us define

$$
\begin{aligned}
\phi_{0}(\eta)=F(\eta, 0)=\mathrm{f}_{0}(\eta), \\
\phi_{k}(\eta)=\left.\frac{1}{k !} \frac{\partial^{k} F(\eta, p)}{\partial p^{k}}\right|_{p=0}(k>0)
\end{aligned}
$$

So that

$$
F(\eta, p)=\phi_{0}(\eta)+\sum_{k=1}^{+\infty} \phi_{k}(\eta) p^{k}
$$

The convergence region of the above series depends upon the linear operator $\mathrm{L}$ and the non-zero parameter $\mathrm{h}$ which are to be selected such that solution converges at $p=1$. Using equation (18) for $p=1$, we get

$$
f(\eta)=\phi_{0}(\eta)+\sum_{m=1}^{+\infty} \phi_{m}(\eta)
$$

where $\phi_{m}$ are unknowns to be determined.

Differentiating $\mathrm{m}$ times the two sides of equations (18) about the embedding parameter $\mathrm{p}$, using Leibnitz theorem, setting $\mathrm{p}=0$ and dividing by $\mathrm{m}$ !, we get

$$
\mathrm{L}\left[\phi_{m}-\chi_{\mathrm{m}} \phi_{\mathrm{m}-1}\right]=\mathrm{hR}_{\mathrm{m}}(\eta)
$$

where

$$
\begin{gathered}
\chi_{m}=\left\{\begin{array}{ll}
0 & \text { when } m \leq 1 \\
1 & \text { when } m>1
\end{array},\right. \\
\mathrm{R}_{\mathrm{m}}(\eta)=\phi_{m-1}^{\prime \prime \prime}(\eta)+\sum_{k=0}^{m-1} \phi_{m-1-k}^{\prime \prime}(\eta) \phi_{k}^{\prime}(\eta)+\sum_{k=0}^{m-1} \phi_{m-1-k}^{\prime}(\eta) \phi_{k}^{\prime}(\eta)-M \phi_{m-1}^{\prime}(\eta)
\end{gathered}
$$

with boundary conditions

$$
\phi_{m}(0)=\phi_{m}{ }^{\prime}(0)=\phi_{m}{ }^{\prime \prime}(0)=\phi_{m}{ }^{\prime}(+\infty)=0 .
$$

From equations (20) we get equations in terms of $\phi_{m}(\eta)$, solving them we get the required solution as $\phi_{0}=\frac{4}{3} \mathrm{e}^{\eta}-\frac{1}{3} \mathrm{e}^{-2 \eta}$

.......so on.

We have

$\mathrm{f}(\eta)=\phi_{0}+\phi_{1}+\phi_{2}+\phi_{3}+\phi_{4}+\phi_{5}+\phi_{6}+\phi_{7}+\phi_{8}+\ldots$ 
The solution $f$ consists of $h$ and is a series solution. To get a valid solution we have to choose $h$ in such a way that the solution series is convergent. The value of $\mathrm{h}$ is obtained by following the method explained by Achala et. al [23, $24,25,26,27]$ where the residual error is calculated and the graph of it verses $\mathrm{h}$ is drawn. When the graph is horizontal then that $\mathrm{h}$ value is considered and with that $\mathrm{h}$ we get a convergent solution. The residual error is defined as follows for kth order HAM solution of $\mathrm{f}$ as,

$$
E_{R}(h)=\frac{1}{\tau_{0}} \int_{0}^{\tau_{0}}\left(f_{\text {exact }}-\sum_{r=0}^{k} f_{k}\right)^{2} d \eta
$$

The graphs of residual error $E_{R}(h)$ versus $h$ for first order, second order, third order, and so on upto sixth order for different are drawn in fig. 1 and the optimal value, $h_{\text {opt }}$ is chosen from these graph which is the value of minimal residual error. The values of optimum $\mathrm{h}$ obtained are listed in Table 1.

We can generate large number of terms (say $n=50$ ) on solving the linear equations by MATHEMATICA. Because of availability of large number of coefficients, we can use Pade's approximation to test the convergence of this series.

\section{Results and Discussions:-}

In this paper we have obtained velocity distribution of two dimensional flow of an incompressible, non-Newtonian, nano fluid flowing steadily under the effect of external magnetic field applied normally to the flow over a shrinking/stretching sheet. The method used is Homotopy Analysis Method (HAM) which is very strong method to solve nonlinear PDE and ODE in series form. This method works for almost all nonlinear flow problems.

In figure 1 we have explained the geometry of flow considered. Figure 2 is a graph to evaluate an important parameter arising in HAM called convergence parameter $\mathrm{h}$, using this estimated value we get convergent series solution. Figure 3 and 3a represent velocity curve for different values of magnetic parameter and $\alpha=0$. In figure

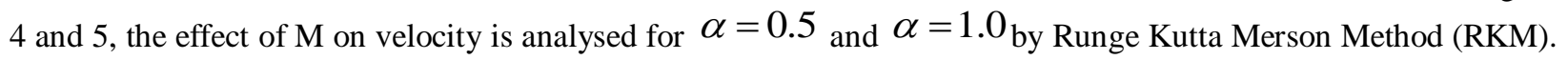
It is observed that HAM and RKM methods show same graphs so Ham solution can be considered as exact solution. In figure 6 and 7 velocity curves are drawn for different $\alpha$ and $M=0$ and $M=2$. Thus we conclude that HAM can be used to find exact solution of given equations.

Further work to analyze the effect of different parameters like Brownian motion, thermophoresis, Prandtle number and Lewis number on the flow and heat transfer is under progress.

\section{Graphs of HAM and RKM Solutions:-}

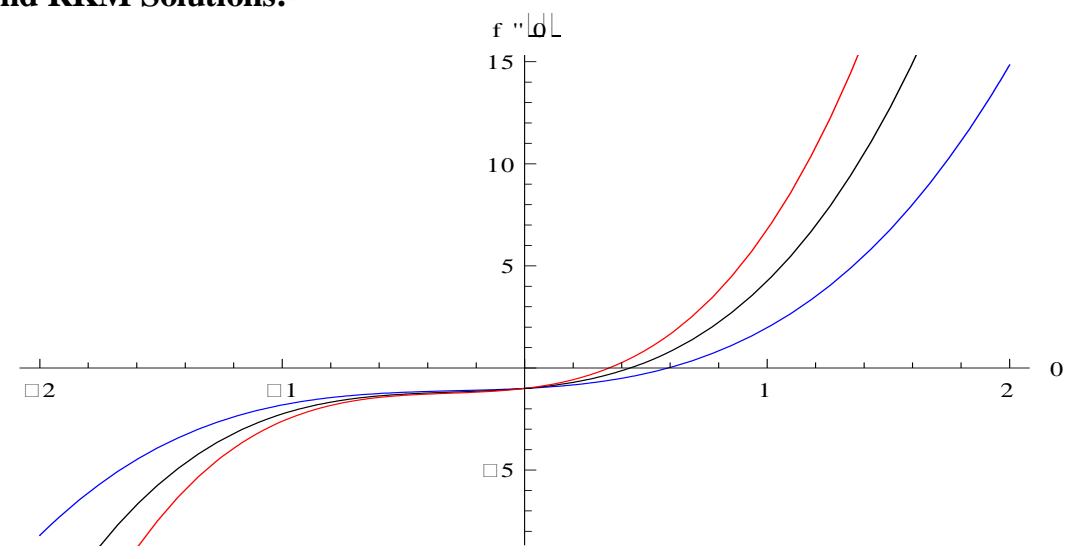

Fig 2:- Graph of f''(0) verses $\eta$ (h curve) 


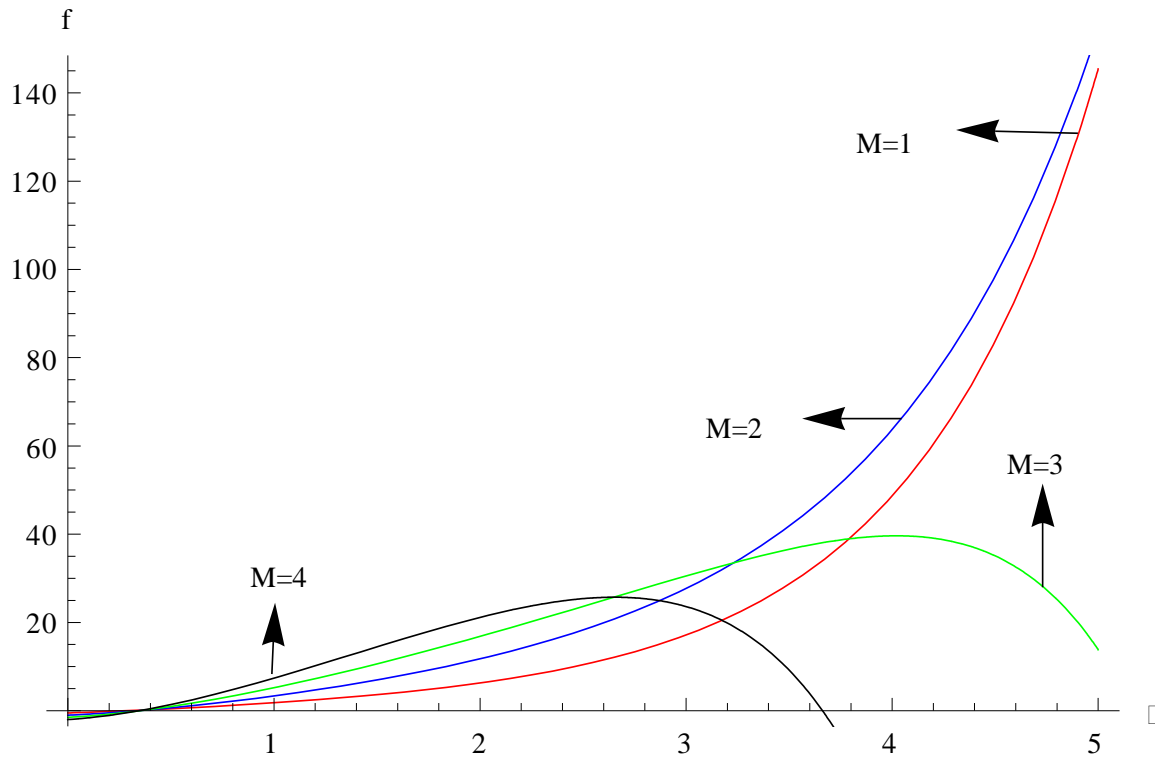

Fig. 3:- Velocity Curve of HAM solution for $\alpha=0$

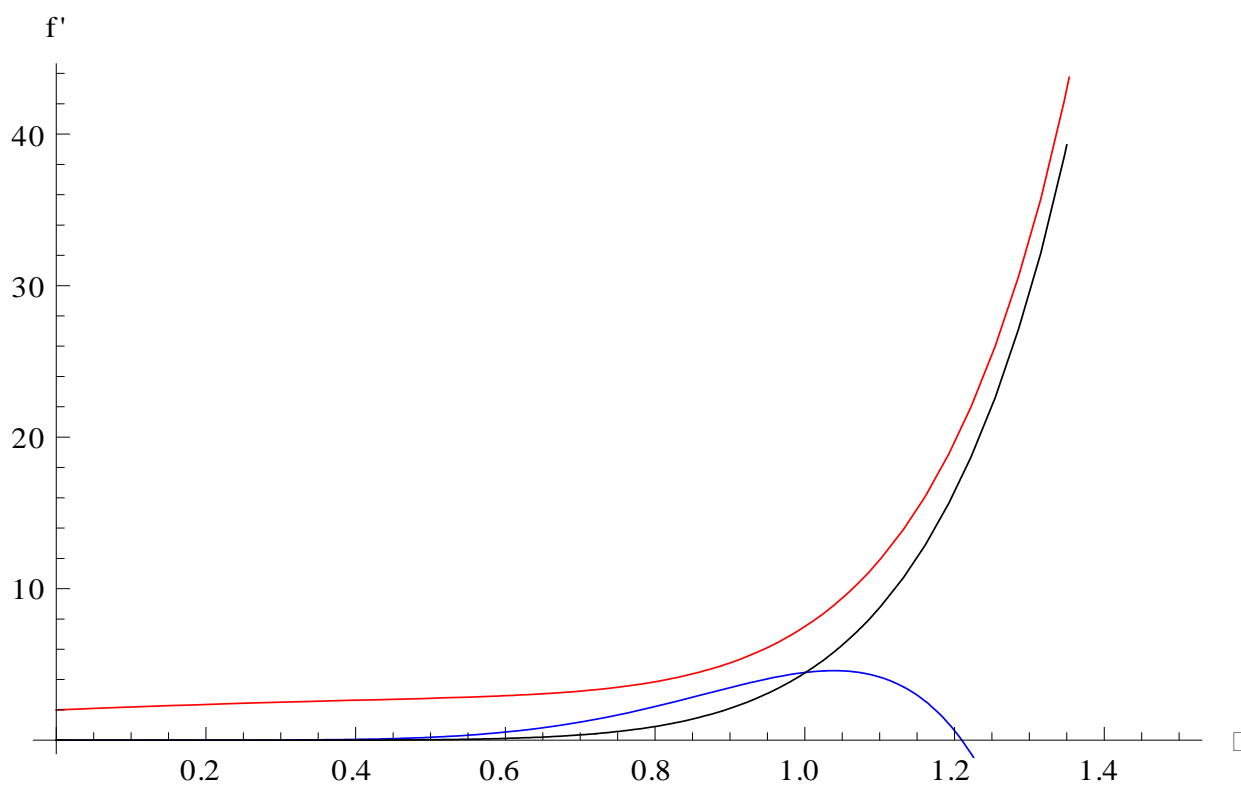

Fig. 3a:- Velocity Curve of Series solution for $\alpha=0$ and $M=2,3,0.5$. 


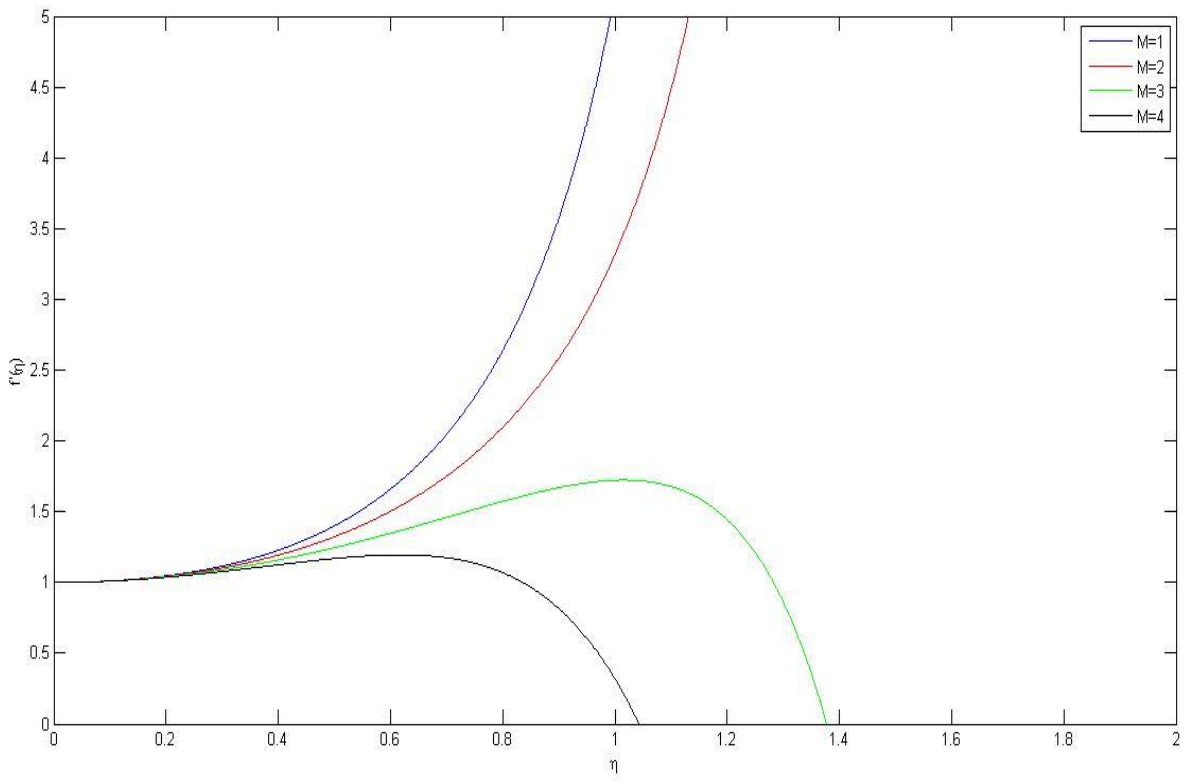

Fig 4:-Velocity Curve for different $\mathrm{M}$ and $\alpha=0.5$ by R-K Merson Method

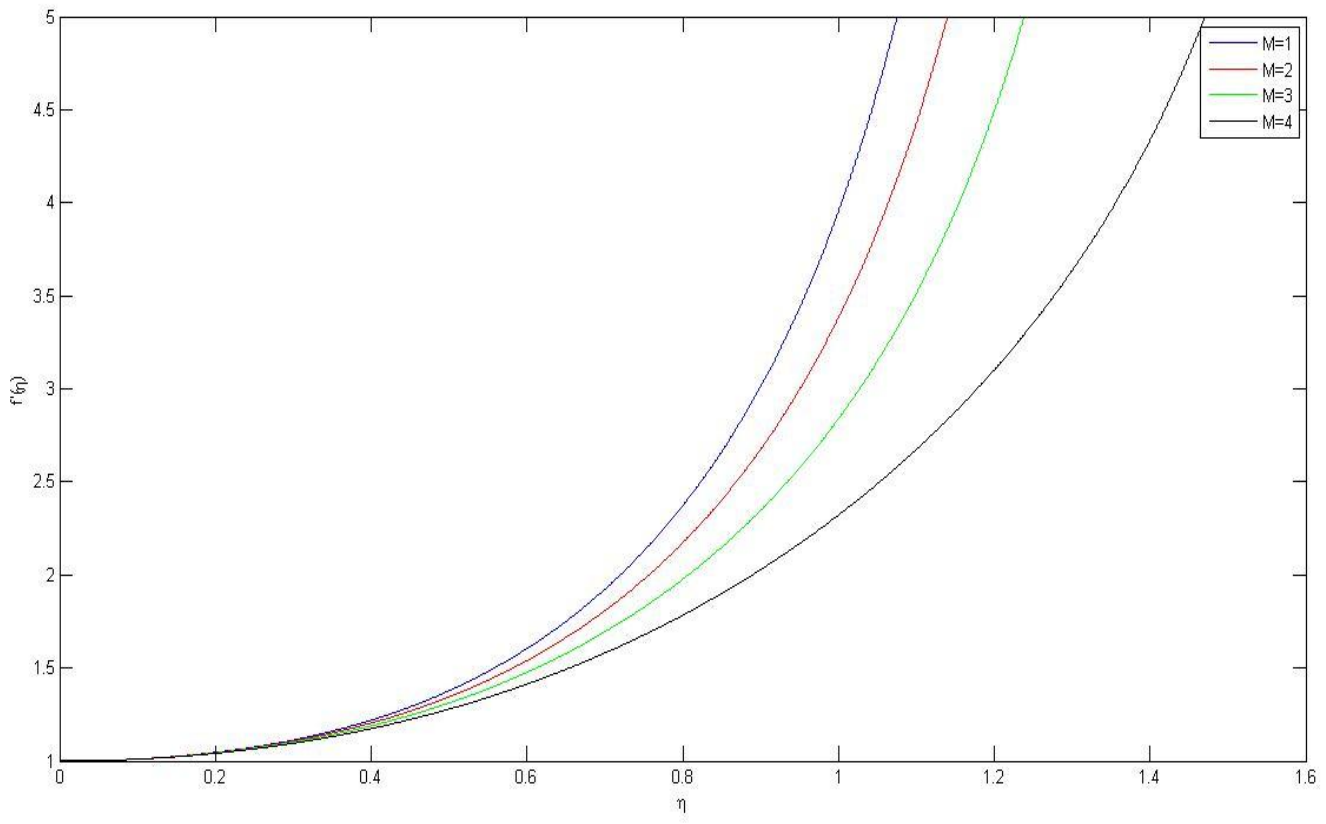

Fig 5:- Velocity Curve for different $M$ and $\alpha=1.0$ by R-K Merson Method 


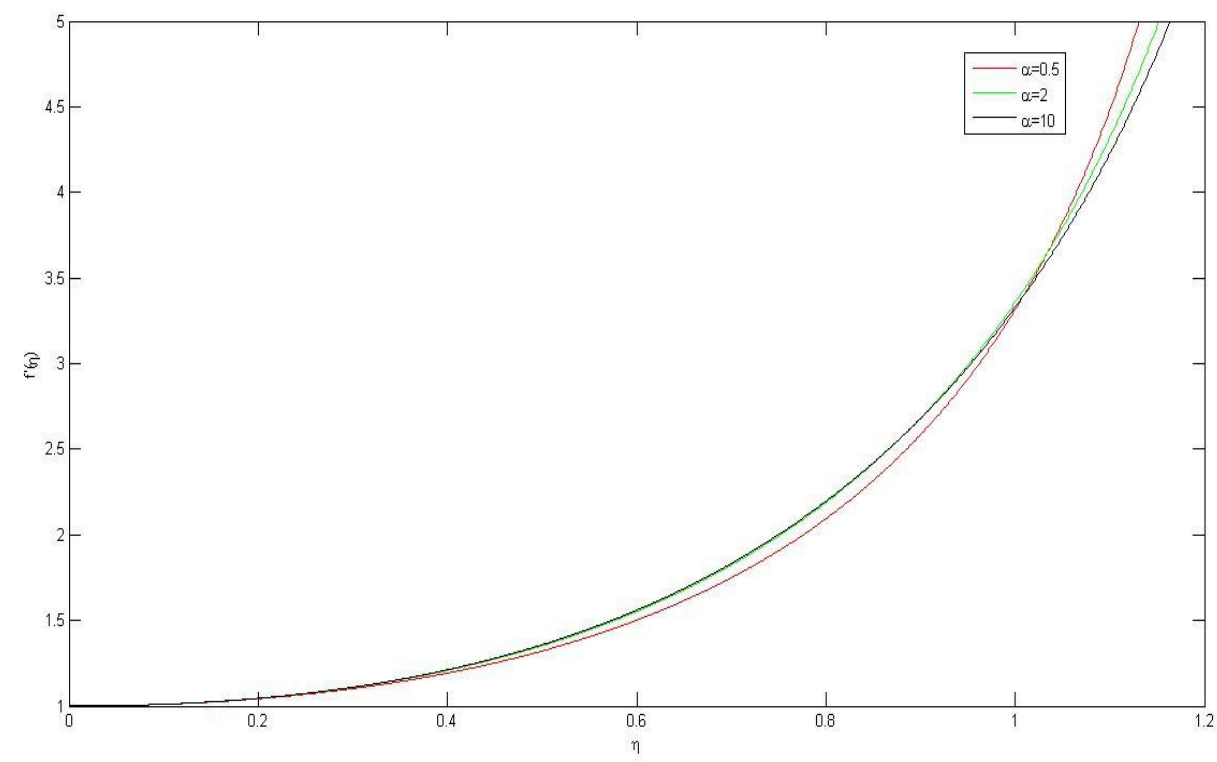

Fig 6:- Velocity Curve for different $\alpha$ and $M=2.0$ by R-K Merson Method

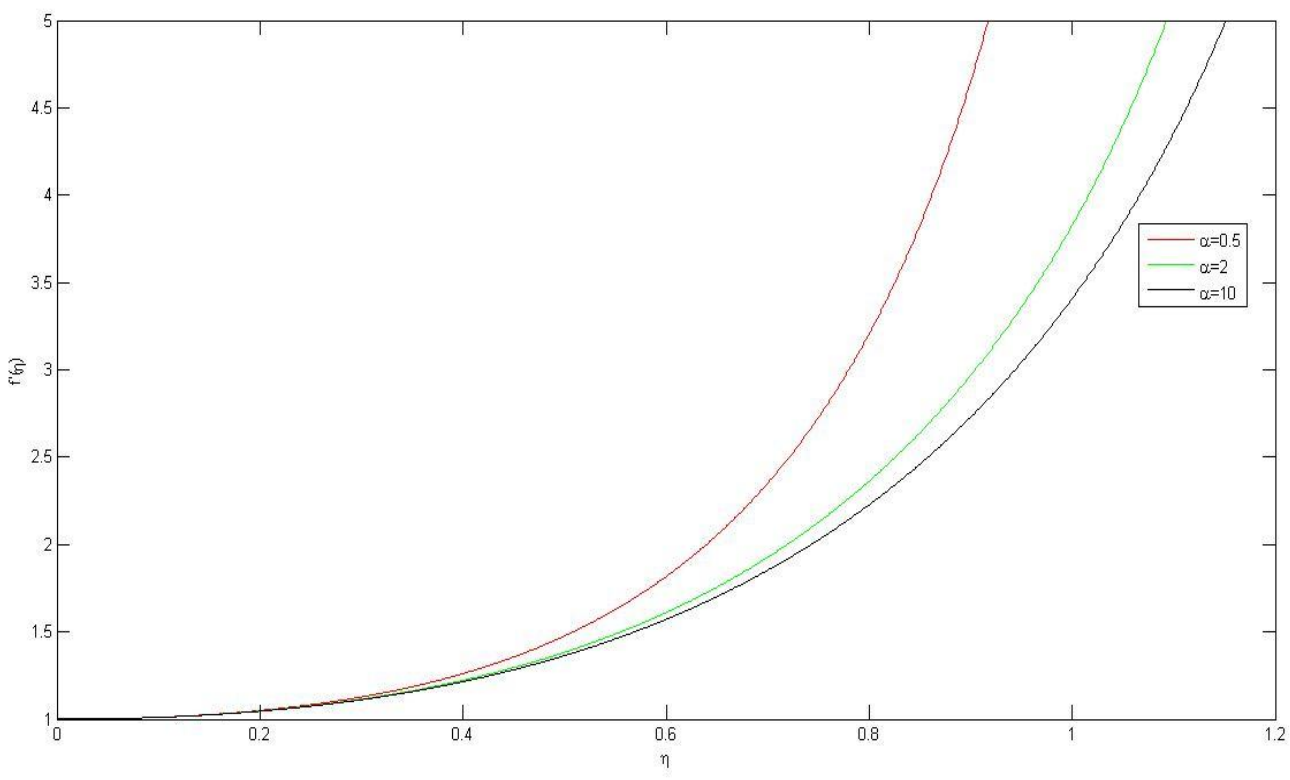

Fig. 7:- Velocity Curve for different $\alpha$ and $M=0$ by R-K Merson Method

\section{Acknowledgement:-}

We are very much thankful to UGC SWRO, Bangalore for providing us with financial assistance to carry out this work through Minor Research Project MRP(s) 702/10-1/KABA031/UGC-SWRO dt.10.02.2011 .

\section{References:-}

1. William Evans, Jacob Fish and Pawel Keblinski (2006) Role of Brownian motion hydrodynamics on nanofluid thermal conductivity, Applied Physics Letters, Vol. 88, pp. 093116(1-3).

2. S. J. Liao (1992) The proposed Homotopy analysis Technique for the solution of Nonlinear problems, $\mathrm{Ph} . \mathrm{D}$ Thesis, Shanghai Tiao Tong University, Shanghai, China. 
3. David Kincaid and Ward Cheney (2010) Numerical Analysis Mathematics of Scientific computing, $3^{\text {rd }}$ Edition, American Mathematical Society, Rhode Island. USA.RKM

4. M. K. Jain (1984) Numerical Solutions of Differential Equations, New Age International Publications, India

5. John. C. Butcher (2008) Numerical Methods for Ordinary Differential Equations, Wiley online Library.

6. Choi. S.U.S (1995) Enhancing thermal conductivity of fluid with nanoparticles. Int. mech engg congress and expositions, Sanfransisco USA, ASME, FED 231/MD, Vol. 66, pp 99-105.

7. Buongiorno. J, (2006) Convective Transport in Nanofluids, Transactions of the ASME, Vol 128, Pp 240250.,2006.

8. Khan W A and Pop I (2010) Boundary layer flow of a nanofluid past a stretching sheet. Int. Jl Heat and Mass transfer Vol.53, pp. 2477-2483.

9. Kuznetsov A V and Nield D A (2010) Natural Convective boundary layer flow of a nanofluid past a vertical plate. Int Jl of thermal Sci, Vol.49, No. 2, pp.243-247.

10. Makinde O and Aziz A (2011) Boundary Layer flow of a nanofluid past a stretching sheet with convective boundary condition. Intl Jl of Thermal Sciences, vol 50, No. 7, pp. 1326-1332

11. Subhas Abel and Monayya Mareppa.(2013) MHD flow and heat transfer of the mixed hydrodynamic / thermal slip over a linear vertically stretching sheet, International Journal of Mathematical Archive, Vol. 4, No. 5, pp. 156-163.

12. K. V. Prasad (2012) Flow and heat transfer at a nonlinearly shrinking porous sheet in a thermally stratified medium, International Journal of Mathematical Archives, Vol. 3, No. 8,pp. 3004-3015.

13. K.V. Prasad, P.S. Datti, K. Vajravelu (2010) Hydromagnetic flow and heat transfer of a non-Newtonian Power law fluid over a vertical stretching sheet, Int. J. Heat and Mass Transfer, Vol.53, No. (5-6), pp.879-888.

14. Hameed M, Nadeem S (2007) Unsteady MHD flow of a non-Newtonian fluid on a porous plate. Jl. Math Anal Appl, Vol. 325. pp. 724-733.

15. P.D. McCormack, and L.J. Crane (1973) Physical Fluid Dynamics, Academic Press, New York.

16. P. S. Gupta and A. S. Gupta (1977) Heat and mass transfer on a stretching sheet with suction and blowing, The Canadian Jl of Chemical Engg, Vol 55, No. 6, pp 744-746.

17. B. K.Dutta P. Roy and A. S Gupta. ( 1985) Temperature field in flow over a stretching sheet with uniform heat flux, Vol 12, No. 1, pp 89-94.

18. C. Y. Wang, (1988) Stretching a surface in a rotating fluid, Jl of Appl Maths and Phy (ZAMP), Vol 39, pp..177-185.

19. C. Y. Wang, (1984) The three dimensional flow due to a stretching flat surface, Phy. Fluids, Vol. 27, pp. 19151917.

20. ] A. Noghrehabadi, R. Pourrajab, and M. Ghalambaz, (2012) Effect of partial slip boundary condition on the flow and heat transfer of nanofluids past stretching sheet prescribed constant wall temperature, Int. J. Therm. Sci., vol. 54, , pp. 253-261.

21. P. Rana and R. Bhargava (2012) Flow and heat transfer of a nanofluid over a nonlinearly stretching sheet a numerical study. Communications in Nonlinear Science and Numerical Simulation, Vol. 17, No.1, pp 212-226.

22. Goyal M and Bhagava R (2014) Boundary layer flow and heat transfer of viscoelastic nanofluid past a stretching sheet with partial slip conditions. Applied Nanosciences, Vol 4, pp.761-767.

23. Achala. L. Nargund and B. Satyanarayana.(2015) Homotopy Analysis method to solve Boussinesq equation, Journal of Applied Physics, Vol. , No., pp. 2015.

24. Achala.L. Nargund and B. Satyanarayan.,: "Approximate analytical solution of MHD compressible boundary layer flow with pressure gradient and suction/injection", Journal of Applied Physics, Vol6. No.1, pg.12161226, 2015.

25. Achala. L. Nargund, B. Satyanarayan and Madhusudan.:" Homotopy Analysis method to solve Boussinesq equation." Journal of Applied Physics, 2015

26. Achala.L. Nargund and B. Satyanarayan (2011) Homotopy Analysis Method for Flow over Nonlinearly Stretching Sheet with Magnetic Field, Jl. of Appl. Math. And Fluid Mech., Vol 3, No.1, pp. 15-22.

27. Achala.L. Nargund and B. Satyanarayan (2013) Homotopy Analysis Method for Nonlinear Boundary Value Problem, JP Jl. Applied Math., Vol. 5, pp. 27-46. 